\title{
DIAGNÓSTICOS E INTERVENÇÕES DE ENFERMAGEM EM UNIDADE DE PRONTO ATENDIMENTO À LUZ DAS NECESSIDADES HUMANAS BÁSICAS
}

Leni de Lima Santanaํㅡ, Verônica de Azevedo Mazza², Samanta Andrine Marschall Taube ${ }^{3}$, Leila Maria Mansano Sarquis ${ }^{4}$

RESUMO: Pesquisa exploratória retrospectiva documental realizada em um Centro Municipal de Urgências Médicas de Curitiba, Paraná cujos objetivos foram identificar as Necessidades Humanas Básicas mais frequentes em pacientes adultos internados e elencar os diagnósticos e intervenções de Enfermagem necessárias ao atendimento das necessidades afetadas. Utilizou-se como base a CIPE® Versão 1, o Projeto CIPESC $®$ e a Teoria das Necessidades Humanas Básicas de Wanda Horta. A coleta de dados ocorreu por meio da análise de 512 documentos que compõem os prontuários dos pacientes. As situações identificadas foram classificadas em 16 necessidades psicobiológicas e uma necessidade psicossocial. Para o atendimento destas foram compostos 214 diagnósticos e 308 intervenções de Enfermagem. A identificação das necessidades afetadas, bem como a elaboração de um plano de assistência coerente com a realidade, são indispensáveis para o alcance de metas de cuidado, sendo o Processo de Enfermagem ferramenta facilitadora do trabalho do Enfermeiro. PALAVRAS-CHAVE: Processos de enfermagem; Diagnóstico de enfermagem; Cuidados de enfermagem; Classificação.

\section{DIAGNOSES AND NURSING INTERVENTIONS IN AN ACCIDENT AND EMERGENCY UNIT IN THE LIGHT OF BASIC HUMAN NECESSITIES}

ABSTRACT: This exploratory, retrospective documental research was undertaken in a Medical Emergency Center in Curitiba, Paraná, Brazil. The study's objectives were to identify the most frequent Basic Human Needs in hospitalized adult patients and to list the diagnoses and nursing interventions necessary to attend the affected needs. ICNP ${ }^{\circledR}$ Version 1, the ICNPCH (International Classification of Nursing Practice - Community Health) Project and Wanda Horta's theory of Basic Human Needs were used as a base. Data collection took place through analysis of 512 documents which made up the patients' medical notes. The situations identified were classified into sixteen psycho-biological need and one psychosocial need. For the situations to be attended, 214 diagnoses were made and 308 nursing interventions. The identification of the needs, and the elaboration of care plans coherent with reality, were indispensable for the achievement of care goals, the Nursing Process being the facilitating tool for the Nursing work. KEYWORDS: Nursing processes; Nursing diagnosis; Nursing care; Classification.

\section{DIAGNÓSTICOS E INTERVENCIONES DE ENFERMERÍA EN UNIDAD DE PRONTO ATENDIMIENTO A LA LUZ DE LAS NECESIDADES HUMANAS BÁSICAS}

RESUMEN: Investigación exploratoria retrospectiva documental realizada en un Centro Municipal de Urgencias Médicas de Curitiba, Paraná, cuyos objetivos fueron identificar las Necesidades Humanas Básicas más frecuentes en pacientes adultos internados y elencar los diagnósticos e intervenciones de Enfermería necesarias al atendimiento de las necesidades afectadas. Se utilizó como base la CIPE® Versión 1, el Proyecto CIPESC $R$ e la Teoría de las Necesidades Humanas Básicas de Wanda Horta. Los datos fueron recogidos por medio del análisis de 512 documentos que componen los prontuarios de los pacientes. Las situaciones identificadas fueron clasificadas en 16 necesidades psicobiológicas y una necesidad psicosocial. Para el atendimiento de estas fueron compuestos 214 diagnósticos y 308 intervenciones de enfermería. La identificación de las necesidades afectadas, así como la elaboración de un plan de asistencia coherente con la realidad, son indispensables para el alcance de metas de cuidado, siendo el Proceso de Enfermería herramienta facilitadora del trabajo del enfermero. PALABRAS-CLAVE: Procesos de enfermería; Diagnóstico de enfermería; Cuidados de enfermería; Clasificación.

*Recorte da Monografia de Conclusão de Curso intitulada "Proposta de Sistematização de Assistência de Enfermagem em um Centro Municipal de Urgências Médicas" apresentada como requisito parcial para conclusão do Curso de Graduação em Enfermagem da Universidade Federal do Paraná-UFPR em 2010.

${ }^{1}$ Enfermeira. Mestranda pelo Programa de Pós-Graduação em Enfermagem-PPGENF UFPR.

${ }^{2}$ Enfermeira. Doutora em Enfermagem. Professora do Curso de Graduação e do PPGENF UFPR. Coordenadora do Grupo de Estudos Família, Saúde e Desenvolvimento-GEFASED.

${ }^{3}$ Enfermeira. Mestre em Enfermagem. Professora do Curso de Graduação em Enfermagem da UFPR. Membro do Grupo de Estudos Multiprofissional em Saúde do Adulto-GEMSA.

${ }^{4}$ Enfermeira. Doutora em Enfermagem. Professora do Curso de Graduação em Enfermagem e do PPGENF UFPR. Membro do GEMSA.

\section{Autor correspondente:}

Leni de Lima Santana

Universidade Federal do Paraná.

R. Maestro Carlos Frank, 2391 - 81750-400 - Curitiba-PR-Brasil

Recebido: 30/09/2011

E-mail: lenisantana@hotmail.com 


\section{INTRODUÇÃO}

Ao longo dos anos a Enfermagem, como profissão, tem procurado um modelo de assistência que atenda o ser humano em todas as suas necessidades (físicas, espirituais e sociais) e que delimite bases científicas e específicas à organização de seu processo de trabalho e, concomitantemente, lhe proporcione visibilidade e credibilidade como ciência ${ }^{(1)}$.

Nesse sentido, o Processo de Enfermagem (PE) é utilizado como um instrumento que orienta, dá suporte às decisões e ao planejamento dos cuidados da Enfermagem, exigindo formulário específico para sua documentação. O PE faz parte da Sistematização da Assistência de Enfermagem (SAE) que, por sua vez, organiza o processo de trabalho da profissão no que se refere à delimitação dos recursos para a operacionalização do PE, devendo ser realizado em todos os ambientes de cuidado profissional ${ }^{(2-3)}$.

O PE é um método sistemático e interrelacionado de prestação de cuidado $^{(2)}$ que envolve toda a equipe de Enfermagem na sua aplicação. É constituído por um conjunto de etapas dinâmicas e interdependentes que, sequencialmente, permitem identificar situações de saúde/doença do indivíduo que embasarão a seleção e a implementação de ações de Enfermagem e o acompanhamento de seus resultados. As etapas para sua execução permitem promover, prever, recuperar e reabilitar a saúde do indivíduo, família e comunidade, sendo elas: coleta de dados, diagnóstico de Enfermagem, planejamento de Enfermagem, implementação e avaliação de Enfermagem $^{(3)}$.

No Brasil, a normatização vigente do Conselho Federal de Enfermagem orienta que o PE esteja embasado em um suporte teórico que sustente a avaliação dos resultados alcançados ${ }^{(3)}$. Sendo assim, destaca-se que um dos modelos existentes é o das Necessidades Humanas Básicas, as quais correspondem às condições ou situações que o indivíduo, família ou comunidade apresentam em função de algum desequilíbrio e que, por isso, necessitam de intervenções da Enfermagem, sendo elas psicobiológicas, psicossociais e psicoespirituais ${ }^{(2)}$.

Para aplicar o PE, o enfermeiro necessita de conhecimento teórico e prático no que diz respeito aos modelos teóricos existentes na Enfermagem, as taxonomias que embasam os diagnósticos, intervenções e resultados, além do uso de acurácia para realizar o julgamento clínico dos dados ${ }^{(4)}$. Além disso, o enfermeiro necessita ser capaz de envolver toda a equipe de Enfermagem na sua realização, ter a clareza dos recursos humanos, físicos e materiais existentes em seu ambiente de trabalho e identificar o perfil da clientela, pois estes se tratam de aspectos fundamentais ao alcance dos resultados estipulados e, consequentemente, a qualidade do cuidado desenvolvido ${ }^{(5)}$.

Destaca-se que quando realizado em instituições prestadoras de serviço ambulatorial de saúde, o PE é usualmente conhecido como consulta de Enferma$\mathrm{gem}^{(6)}$. Um destes ambientes é o Centro Municipal de Urgências Médicas (CMUM), local que difere tanto de Unidades Básicas de Saúde, como daquelas com Estratégia Saúde da Família, ou ainda, de serviços hospitalares, constituindo-se como novo cenário de atuação do enfermeiro.

Espaço construído como resultado da Política Nacional de Atenção às Urgências, instituída pelo Ministério da Saúde em parceria com os Estados e Municípios, o CMUM possui como objetivo ampliar, aperfeiçoar e implementar as redes regionalizadas e hierarquizadas dos serviços de saúde, contribuindo com a organização e qualidade do atendimento em urgência e emergência ${ }^{(7)}$.

O CMUM se caracteriza por apresentar alta demanda e rotatividade de atendimento em saúde, sendo que o perfil da clientela revela quadros diversos que variam em sua complexidade ${ }^{(8)}$. Assim, nesse espaço, o enfermeiro tem por desafio direcionar as suas ações para promover atendimento efetivo, de forma ágil e individualizado, o qual deve ser subsidiado pela utilização do PE.

Considerando a importância do PE para o atendimento em saúde de qualidade em todos os seus níveis, o que inclui o atendimento de urgência e emergência, comprometendo-se que este sirva como instrumento ao embasamento das ações do enfermeiro, além de contribuir para a visibilidade e autonomia do cuidado desenvolvido por este profissional. Isto posto, os objetivos deste estudo foram identificar as Necessidades Humanas Básicas (NHB) mais frequentes nos indivíduos internados em um Centro Municipal de Urgências Médicas de Curitiba (PR) e identificar os diagnósticos e intervenções de Enfermagem necessários ao atendimento das NHB afetadas.

\section{METODOLOGIA}

Trata-se de uma etapa do Projeto de Pesquisa intitulado "Sistematização da Assistência de En- 
fermagem em um Centro Municipal de Urgências Médicas". Neste recorte, utilizou-se o tipo de estudo exploratório, documental retrospectivo, de abordagem quantitativa, cujos dados foram coletados no período de junho a novembro de 2009.

Utilizou-se para a coleta de dados 512 documentos, sendo que 138 correspondiam aos registros médicos e 374 as Fichas de Prescrição de Enfermagem escolhidos a partir dos seguintes critérios de inclusão: ter gerado Autorização para Internação Hospitalar (AIH) e pertencerem a pacientes com idade superior a 18 anos, internados entre agosto e novembro de 2008 no local de estudo.

As fichas de Enfermagem eram instrumentos institucionalizados pela Secretaria Municipal de Saúde de Curitiba e utilizados diariamente para registrar o processo de trabalho da equipe de Enfermagem. As fichas faziam parte do prontuário do usuário e compostas por: identificação do paciente; um campo com 13 intervenções de Enfermagem padronizadas pelo serviço e espaço para a inclusão de outras, conforme o profissional julgasse necessário; um campo para a evolução e anotação de Enfermagem; e um quadro para os controles de sinais vitais, ingestas e eliminações do paciente.

Para o desenvolvimento e alcance dos objetivos propostos, realizaram-se 4 etapas. No primeiro momento, fez-se uma análise dos documentos, por meio de leitura minuciosa dos 512 documentos selecionados, identificando os sinais, os sintomas e as ações de cuidado registradas pelos diferentes profissionais da equipe, bem como a anotação de frequência de cada um deles, no intuito de elencar as pistas diagnósticas que auxiliariam na identificação das NHB comprometidas. Os dados foram descritos quantitativamente e apresentados no formato de quadros e tabelas.

No segundo momento, houve a correlação das pistas diagnósticas com as necessidades psicobiológicas, psicossociais e psicoespirituais, conforme previstas pelas NHB de Wanda Horta ${ }^{(2)}$. Para sua realização foram utilizados conceitos da Teoria em questão, bem como a experiência profissional das pesquisadoras.

No terceiro momento, houve a elaboração dos Diagnósticos de Enfermagem a partir da correlação entre as pistas diagnósticas e as NHB afetadas. A seguir, procedeu-se a correlação destas com as definições de termos relativos aos fenômenos de Enfermagem, contidos nos Eixos Foco e Julgamento da CIPE $^{\circledR}$ Versão $1.0^{(9)}$, e com alguns diagnósticos pertencentes ao prontuário eletrônico da rede municipal de saúde, os quais têm por base a taxonomia CIPESC $^{\circledR(10)}$.

No quarto momento, houve a determinação das Intervenções de Enfermagem, utilizando-se as diretrizes do Conselho Internacional de Enfermagem (CIE) que determinam a inclusão obrigatória de termos dos Eixos Foco e Ação, com a adição de outros eixos conforme a necessidade ${ }^{(9)}$. Para as intervenções levou-se em consideração os diagnósticos elencados no momento anterior. O raciocínio clínico, a acurácia diagnóstica e a experiência das pesquisadoras também foram relevantes nesta etapa.

O estudo considerou as determinações da Resolução 196/96 do Conselho Nacional de Saúde e a pesquisa foi aprovada pelo Comitê de Ética e Pesquisa do Setor de Ciências da Saúde da Universidade Federal do Paraná sob CEP n.647.184.08.11.

\section{RESULTADOS}

A leitura dos documentos selecionados permitiu identificar 2.514 registros de situações adversas apresentadas pelos pacientes as quais variaram de $1 \mathrm{a}$ 145 tipos de registros, constituindo, assim, 132 pistas diagnósticas. A correlação das pistas diagnósticas com os conceitos de NHB, estabelecidos por Wanda Horta $^{(2)}$, resultou na identificação de 15 necessidades psicobiológicas e uma necessidade psicossocial, necessidades psicoespirituais não foram identificadas na amostra.

A partir da identificação das NHB afetadas foram determinados 214 diagnósticos voltados ao atendimento das mesmas. Considerando a prioridade na prestação de cuidado e a relevância da necessidade afetada ${ }^{(11)}$ o número de diagnósticos variou de $44(20,56 \%)$ para a necessidade de Oxigenação à cinco $(2,33 \%)$ para Sono e Repouso e Cuidado Corporal.

Dentre as necessidades psicobiológicas destacaram-se a Regulação Vascular com $15,91 \%$ das pistas diagnósticas identificadas e Eliminação com 14,40\%. As necessidades desta categoria, que apresentaram menor frequência de pistas diagnósticas, foram Sono e Repouso, Cuidado Corporal, Regulação Térmica, Regulação Imunológica e Regulação Hormonal com apenas $1,51 \%$ cada. A necessidade psicossocial Segurança foi a terceira maior situação identificada, representando $12,12 \%$ do total.

Foram determinadas 308 intervenções de En- 
fermagem para os diagnósticos identificados. A frequência destas variou de $12,98 \%$ ao atendimento da necessidade de Eliminação e $2,27 \%$ para a necessidade de Sono e Repouso. No que se refere à ação utilizada para o cuidado, $132(42,85 \%)$ correspondem a determinar; $68(22,07 \%)$ a desempenhar, ou seja, realizar uma tarefa; $46(14,93 \%)$ a administrar, que se refere ao ato de distribuir alguma coisa, providenciar, aplicar; 38 (12,33\%) à ação de informar e 24 (7,79\%) à ação de atender, preocupar-se, tomar conta de alguém ou de alguma coisa ${ }^{(9)}$.

A partir dos resultados, quadros foram elaborados para correlacionar os Diagnósticos de Enfermagem (com suas pistas) com as Intervenções de Enfermagem. Embora 16 quadros tenham sido elaborados no estudo, a apresentação dos mesmos neste recorte restringiu-se àqueles para os quais se obteve a maior frequência de pistas identificadas em sua categoria: necessidade psicobiológica de Regulação Vascular e necessidade psicossocial de Segurança.

As pistas diagnósticas de maior relevância para a necessidade de Regulação Vascular foram edema
$(16,4 \%)$, crise hipertensiva $(12,4 \%)$ e hipotensão $(6,8 \%)$. A parada cardiorrespiratória teve $0,4 \%$ de frequência, tendo sido citada apenas uma vez nos documentos avaliados. Assim, elaborou-se um quadro com os Diagnósticos e Intervenções de Enfermagem CIPE $^{\circledR}$ e CIPESC ${ }^{\circledR}$ (Quadro 1).

As principais pistas diagnósticas identificadas para a necessidade psicossocial Segurança relacionaram-se aos distúrbios decorrentes de transtornos psiquiátricos. Desta forma, foram evidenciadas 16 situações, com prevalência da agitação com $32,22 \%$ dos casos, crise depressiva e etilismo, com $8,9 \%$ cada.

O plano de cuidados direcionados ao atendimento desta necessidade previu: suporte à proteção contra danos; oferecer ajuda imediata em crises psicológicas e fisiológicas; reduzir os riscos e manter monitoração contínua dos mesmos ${ }^{(12)}$. Partindo deste princípio, e considerando as pistas diagnósticas identificadas, o conhecimento empírico das pesquisadoras e obedecendo aos critérios estabelecidos pelo referencial CIPE ${ }^{\circledR}$ e CIPESC $^{\circledR}$, foi elaborado o quadro a seguir (Quadro 2).

Quadro 1 - Diagnósticos e Intervenções de Enfermagem para a Necessidade Psicobiológica de Regulação Vascular. Curitiba, 2009

\begin{tabular}{|ll|}
\hline \multicolumn{2}{|c|}{ Necessidades psicobiológicas de Regulação Vascular - CIPE ${ }^{\circledR}$ Versão 1 } \\
\hline Diagnósticos de Enfermagem & Intervenções de Enfermagem \\
- Bradicardia & - Apoiar o paciente e a família \\
- Débito cardíaco diminuído & - Orientar o paciente quanto à dieta hipossódica e hipogordurosa \\
- Edema atual em (citar localização) & - Avaliar uso de meia elástica \\
- Edema em nível elevado em (citar localização) & - Controlar volume de líquidos infundidos \\
- Edema leve em (citar localização) & - Diminuir a ansiedade do paciente \\
- Edema moderado em (citar localização) & - Elevar pernas \\
- Frequência cardíaca alterada & - Evitar fatores de stress ao paciente \\
- Perfusão tissular alterada & - Instalar e monitorar oxigenoterapia prescrita \\
- Pressão sanguínea alterada & -Investigar,registrarecomunicaraomédico/enfermeira queixadedor \\
- Processo vascular alterado & - Manter em monitor cardíaco \\
- Risco aumentado para edema & - Manter paciente em repouso \\
- Ritmo cardíaco alterado & - Monitorar padrão respiratório \\
- Sistema cardiovascular comprometido & - Monitorar perfusão tissular \\
- Taquicardia & - Monitorar resposta ao tratamento \\
& - Monitorar sinais vitais \\
& - Monitorar estado neurológico \\
& - Tranquilizar o paciente \\
& - Verificar frequência cardíaca de $2 / 2 \mathrm{~h}$ \\
& - Verificar frequência de pulso de $2 / 2 \mathrm{~h}$ \\
& - Verificar pressão arterial de $2 / 2 \mathrm{~h}$ \\
\hline
\end{tabular}

Cogitare Enferm. 2011 Out/Dez; 16(4):675-81 
Quadro 2 - Diagnósticos e Intervenções de Enfermagem para a Necessidade Psicossocial de Segurança. Curitiba, 2009

\begin{tabular}{|ll|}
\hline \multicolumn{3}{|c|}{ Necessidades psicossociais de segurança - CIPE ${ }^{\circledR}$ Versão 1 } \\
Diagnósticos de Enfermagem & Intervenções de Enfermagem \\
\hline - Alucinação & - Avaliar resposta à medicação \\
- Ansiedade excessiva & - Avaliar sinais de comprometimento psicológico \\
- Ansiedade moderada & - Avaliar status neurológico \\
- Ansiedade & - Avaliar status psicológico \\
- Comportamento agressivo & - Controlar agitação \\
- Depressão & - Conversar com o paciente \\
- Medo & - Elevar as grades do leito \\
- Negação & - Elevar o tórax para evitar aspiração \\
- Risco de trauma & - Estimular a autoestima \\
- Risco para isolamento social & - Estimular a adesão ao tratamento \\
- Uso excessivo de álcool & - Estimular o autocuidado \\
& - Evitar ruídos e stress \\
& - Explicar as rotinas do serviço \\
& - Explicar complicações decorrentes do uso de álcool \\
& - Explicar complicações decorrentes do uso de drogas \\
& - Imobilizar o paciente se apresentar agitação \\
& - Imobilizar pernas e braços se houve agitação \\
& - Incentivar a socialização \\
& - Incentivar os familiares a apoiar o paciente na aderência ao tratamento \\
& - Investigar depressão \\
& - Manter vigilância contínua \\
& - Mobilizar o paciente de $2 / 2 \mathrm{~h}$ \\
& - Monitorar sinais vitais \\
& - Proibir o uso de álcool em ambiente hospitalar \\
& - Tranquilizar o paciente \\
\hline
\end{tabular}

\section{DISCUSSÃO}

As NHB afetadas dos pacientes internados no CMUM são muitas e variadas, conforme evidenciou o estudo. no entanto, discutiremos apenas as necessidades psicobiológicas de Regulação Vascular e Eliminação e a psicossocial Segurança por serem as que apresentaram o maior número de pistas diagnósticas e pelo fato de que a discussão dos resultados na íntegra foge ao foco do presente artigo.

Na definição de Horta, as NHB são estados de tensões conscientes ou inconscientes, resultantes de desequilíbrios hemodinâmicos dos fenômenos vitais cuja intensidade varia dependendo do desequilíbrio instalado. São condições que sofrem interferências de diversos fatores, como idade, sexo, cultura, ciclo saúde-enfermidade, entre outros exigindo resolução sempre que instaladas ${ }^{(2)}$.
No local do estudo, a Regulação Vascular foi a que apresentou o maior número de pistas diagnósticas nos documentos analisados, demonstrando a relação entre esta necessidade afetada e as doenças do aparelho circulatório, as quais estão entre as patologias mais frequentes nos pacientes internados no $\mathrm{CMUM}^{(13)}$. Estes achados correspondem, na atualidade, às problemáticas de doenças vasculares que estão entre as principais causas de morte na população adulta, em todo o mundo. Dentre elas, a hipertensão arterial atinge de 20 a $30 \%$ da população adulta, tendo a crise hipertensiva como uma de suas principais complicações, caracterizada por aumento abrupto, intenso e sintomático da pressão arterial, podendo causar deterioração dos órgãos-alvo ${ }^{(14)}$.

Para a avaliação do sistema vascular, o Enfermeiro precisa estar familiarizado com a anatomia e fisiologia 
do coração, bem como com os sinais e sintomas apresentados quando este órgão estiver afetado. Dentre os fatores a serem observados destacam-se: avaliação dos pulsos apicais e periféricos; e identificação de fadiga, edema, dor torácica, estase jugular e alterações na pressão arterial ${ }^{(15)}$. Através de avaliação correta do paciente, bem como da identificação da necessidade afetada, o enfermeiro terá subsídios indispensáveis para o desenvolvimento de plano de cuidados condizente e capaz de suprir necessidades.

A respeito da NHB de Eliminação, compreendese como uma capacidade que o organismo possui de livrar-se de substâncias indesejáveis ou presentes em quantidade excessiva ${ }^{(16)}$. As pistas diagnósticas nesta necessidade referiram-se, principalmente, a problemas urinários. De acordo com a Sociedade Brasileira de Infectologia, as infecções do trato urinário apresentam alta incidência na população idosa e cerca de 15 a $20 \%$ dos pacientes hospitalizados são submetidos à sondagem vesical de demora por períodos curtos, aumentando, assim, a incidência de infecção urinária ${ }^{(12)}$.

A idade dos pacientes internados no CMUM variou de 19 a 92 anos, sendo que a faixa etária predominante foi de pacientes com idade igual ou superior a 65 anos, ou seja, pessoas mais susceptíveis a problemas urinários. O tempo médio de internação é de 2,67 dias ${ }^{(13)}$, reforçando, assim, os dados da Sociedade Brasileira de Infectologia de incidência de doenças urinárias em pacientes submetidos à sondagens vesicais.

Os sinais e sintomas indicativos de Necessidade de Eliminação afetada incluem mudanças na frequência, odor, queixas de dor, hábitos, sangramentos, consistência e/ou aparência das eliminações, bem como o uso de cateteres e sondagens ${ }^{(2,15)}$. Os dados obtidos devem ser utilizados pelo Enfermeiro com o intuito de planejar cuidadosamente a assistência de Enfermagem, utilizando diagnósticos que atendam as necessidades de cuidado e intervenções voltadas ao estabelecimento e manutenção dos padrões normais, bem como controlar as complicações resultantes dos padrões alterados ${ }^{(12)}$.

Para a análise da necessidade psicossocial Segurança foram consideradas as diretrizes do CIPESC ${ }^{\circledR}$, eo conhecimento empírico das pesquisadoras, uma vez que o referencial teórico utilizado não possui características definidoras que auxiliem na determinação dos sinais e sintomas pertencentes a cada necessidade. Para tanto, foram consideradas as situações que, de alguma forma, pudessem contribuir para o comprometimento da segurança física e psicológica do próprio paciente, assim como para a segurança de funcionários e de terceiros. Dessa forma, as principais pistas diagnósticas identificadas para esta necessidade foram relacionadas aos distúrbios decorrentes de transtornos psiquiátricos.

Os distúrbios psiquiátricos constituem a terceira maior causa de internamento no CMUM. O etilismo, a intoxicação alcoólica, a tentativa de suicídio e a dependência química correspondem a $81 \%$ dos casos desta categoria ${ }^{(13)}$.

\section{CONCLUSÕES}

A identificação das NHB afetadas e a elaboração de plano de assistência são aspectos indispensáveis para a sistematização e organização do serviço de Enfermagem em atendimento integral, eficiente e de qualidade em todos os ambientes de cuidado, incluindo-se o CMUM. Neste contexto, a aplicação do PE facilita e direciona o trabalho do Enfermeiro, pois é através dele que se identificam as necessidades de cuidado e planejamento de ações direcionadas a minimizar ou solucionar os problemas apresentados.

A CIPE ${ }^{\circledR}$ oferece subsídios para a Sistematização da Assistência de Enfermagem uma vez que permite estabelecer diagnósticos e intervenções para pacientes com diversos quadros clínicos e de diferentes áreas de cuidado, além de facilitar a documentação das ações e avaliar a qualidade da assistência.

A adoção do $P E$ e da CIPE $^{\circledR}$, instrumentos que favorecem a autonomia profissional e a tomada de decisões, contribuem para a visibilidade profissional e para a ciência da Enfermagem. Para tanto, entende-se que os profissionais precisam deixar de realizar ações baseadas apenas na avaliação e no diagnóstico médico e passar a atuar fundamentados também em conhecimento construídos pela própria profissão, avaliando, identificando as necessidades de cuidado e intervindo de forma criteriosa e consciente para a solução das mesmas.

Acredita-se que a identificação de necessidades, bem como de diagnósticos e intervenções emergindo da prática, colabora com a identificação das reais necessidades de cuidado do paciente, assim como promove melhor aceitação destes por parte dos profissionais, uma vez que pode ser percebido como algo intrínseco ao processo de trabalho.

Apesar das vantagens apontadas com a utilização das NHB e da CIPE ${ }^{\circledR}$ no presente estudo, algumas dificuldades emergiram. Em relação às NHB, a produção científica consultada não apresenta definição de cada necessidade e nem os sinais e sintomas que as caracterizam. Dificuldade semelhante ocorre com os fenômenos de Enfermagem da CIPE ${ }^{\circledR}$ que não apresenta características definidoras, dificultando o raciocínio diagnóstico. 
Por fim, espera-se que este estudo contribua para a melhoria na qualidade da assistência de Enfermagem em serviços de pronto atendimento, que instigue os profissionais a empregar o raciocínio clínico na avaliação do paciente, e que indique novos caminhos a trilhar na utilização do PE. Contudo, os termos utilizados, bem como os diagnósticos e as intervenções compostas, são passíveis de revisão, adaptações e modificações, de forma que atendam às exigências nacionais e internacionais da categoria e contribuam para o desenvolvimento da profissão.

\section{REFERÊNCIAS}

1. Westphalen MEA, Carraro TE. Metodologia para a assistência de enfermagem: Teorizações, modelos e subsídios para a prática. Goiânia: AB; 2001.

2. Horta WA. O processo de enfermagem. São Paulo: EPU/ EDUSP; 1979.

3. Conselho Federal de Enfermagem. Resolução ${ }^{\circ}$ 358/2009. Dispõe sobre a Sistematização da Assistência de Enfermagem - SAE - nas instituições de saúde brasileiras. Rio de Janeiro: COFEN; 2009.

4. Matos FGOA, Cruz DALM. Construção de instrumento para avaliar a acurácia diagnóstica. Rev Esc Enferm. 2009;43(n. esp):1088-97.

5. Hermida PMV. Araújo IEM. Sistematização da assistência de enfermagem: subsídios para a implantação. Rev Bras Enferm. 2006;59(5):675-9.

6. Luciana MR, Nen Nalú AM, Silvana RM, Vera R. A consulta de enfermagem no cuidado à pessoa com câncer: contextualizando uma realidade. Cogitare Enferm. 2007;12(4):487-93.

7. Ministério da Saúde (BR). Política Nacional de Atenção às Urgências. $3^{a}$ ed. ampliada, Série E. Legislação em Saúde. Brasília; 2006.

8. Secretaria Municipal de Saúde [Internet]. Curitiba: Secretaria Municipal de Saúde; [acesso em 27 jul 2009]. Histórico da Secretaria Municipal de Saúde. Disponível: http://www.curitiba.pr.gov.br/saude.

9. Conselho Internacional de Enfermeiras. Classificação Internacional para a Prática de Enfermagem - CIPE® Versão 1. São Paulo: Algol; 2007.

10. Albuquerque LM, Cubas MR, Martins SK. Nomenclatura de diagnósticos e intervenções de enfermagem da rede básica de saúde do município de Curitiba. In: Albuquerque LM, Cubas MR, organizadoras. Cipescando em Curitiba: construção e implementação de diagnósticos e intervenções de enfermagem na rede básica de saúde. Curitiba: ABEn-Seção-PR; 2005.

11. Bub MBC, Garcia TR. Necessidades humanas: do mito à enfermagem atual. In: Garcia TR. Egry EY, organizadoras. Integralidade da atenção no SUS e Sistematização da Assistência de Enfermagem. Porto Alegre: Artmed; 2010. p. 41-62.

12. McCloskey JC, Bulechek MG. Classificação das intervenções de enfermagem - NIC. $3^{\mathrm{a}}$ ed. Porto Alegre: Artmed; 2004.

13. Souza C. Sistematização da assistência de enfermagem em um centro de urgências médicas: percepção dos enfermeiros [monografia]. Curitiba(PR): Universidade Federal do Paraná; 2009.

14. Martin JFV, Higashiama E, Garcia E, Luizon MR, Cipullo JP. Perfil da crise hipertensiva. Prevalência e apresentação clínica. Arq. Brasileiros de Cardiologia. [Internet] 2004; 83(2) [acesso em 12 nov 2009]. Disponível: http://abc. cardiol.br/2004/8302/83020004.pdf.

15. Potter P. Semiologia em enfermagem [tradução da $4^{\mathrm{a}}$ ed. original]. Rio de Janeiro: Reichmann \& Affonso; 2002.

16. Benedt SA, Bub MBC. Manualde diagnósticos de enfermagem: uma abordagem baseada na Teoria das Necessidades Humanas Básicas e na classificação diagnóstica da NANDA. Florianópolis: Bernúncia, 1998. 